\title{
A comparative study of perineal morbidity in vaginal delivery with and without episiotomy
}

\author{
Priyankur Roy ${ }^{1}$, Sujatha M.S. ${ }^{1}$, Bivas Biswas $^{2}$, Anumita Chatterjee ${ }^{1}$, Pijushkanti Roy ${ }^{3}$
}

\author{
${ }^{1}$ Department of Obstetrics \& Gynaecology, JSS Hospital, Mysore, Karnataka, India \\ ${ }^{2}$ Department of Obstetrics \& Gynaecology, King's Mill Hospital, Sutton in Ashfield, Nottinghamshire, U.K. \\ ${ }^{3}$ Department of Obstetrics \& Gynaecology, Roy's Clinic, Siliguri, West Bengal, India
}

Received: 08 August 2015

Revised: 04 September 2015

Accepted: 08 September 2015

\author{
*Correspondence: \\ Dr. Priyankur Roy, \\ E-mail: priyankurroy@gmail.com
}

Copyright: $\odot$ the author(s), publisher and licensee Medip Academy. This is an open-access article distributed under the terms of the Creative Commons Attribution Non-Commercial License, which permits unrestricted non-commercial use, distribution, and reproduction in any medium, provided the original work is properly cited.

\begin{abstract}
Background: The objective was to determine the occurrence of perineal morbidity in women who delivered vaginally with an episiotomy versus those who delivered without.

Methods: Primigravid women were enrolled into the study on documentation of full dilatation of the cervix and randomized into either the study group (delivered without an episiotomy) or Control Group (delivered with an episiotomy). All labours were carefully monitored with intermittent auscultation of foetal heart rate and partograph was plotted for everyone. Labour was augmented with oxytocin infusion if required. Good perineal and para urethral support at the time of crowning of the head and during delivery of the baby was given for patients of both the groups. Right mediolateral episiotomy was given for the control group. Perineum was then examined and if any lacerations were noted it was sutured, if necessary, as per standard protocol (vicryl rapide was used). Episiotomy was sutured in 3 layers with vicryl rapide.

Results: The total number of patients studied was 300 - equally distributed in both the groups. The age group of the patients and birth weight of the babies were comparable. In the study group, $22.0 \%$ patients had no lacerations in the perineum. Inspite of an episiotomy, $15.34 \%$ had anterior and posterior perineal lacerations and in that $4.67 \%$ patients had anal sphincter tear. 9 patients had more than $50 \%$ of EAS torn but none of them complained of incontinence during their follow-up. $68.37 \%$ patients in the control group had persistent perineal pain for more than one week versus $19.42 \%$ in the study group. $4.27 \%$ patients in the control group had persistent pain whereas no patients in the study group had pain more than 2 months.

Conclusions: Short term perineal morbidity is significantly lower in parturients who delivered without an episiotomy and that episiotomy did not offer protection against sustaining severe perineal lacerations.
\end{abstract}

Keywords: Restricted episiotomy, Universal episiotomy, Perineal dysfunction.

\section{INTRODUCTION}

Perineal trauma is a common event affecting up to $90 \%$ of first time mothers. ${ }^{1}$ It is a cause for concern for many women and in some countries has led to a large increase in the number of women requesting for elective caesarean sections. In the metro cities in India, forty-seven percent of births are by caesarean section and the main contributing factor in the alarming increase in caesarean section rates could be private sector care. ${ }^{2}$ Considerable postnatal morbidity and occasionally mortality can be attributed to this.

Various etiological factors have been associated with perineal trauma namely, large infants, prolonged $2^{\text {nd }}$ stage, instrumental delivery, race, ethnicity and 
nutritional status. Perineal trauma can occur either spontaneously or subsequent to episiotomy.

Perineal lacerations are avoided by preventing too rapid an expulsion of the head (by promoting flexion of the head - Ritgen Manouver) and delivery of the head in between contractions. During crowning, the palmar surface of fingers of one hand is placed over the vertex to prevent the head from being born during a contraction and the other hand is placed over the perineum to help ease the vertex out of the vulval outlet. Episiotomy is given if indicated like in rigid perineum, malpositions, malpresentations (breech), instrumental deliveries, big baby, VBAC, preterm delivery, heart disease, PIH, IUGR and multi-foetal gestation.

The role of episiotomy of late, in normal vaginal delivery, which was once thought to protect the perineum, is being questioned world-wide. ${ }^{3}$

This study was, therefore, planned to assess the perineal morbidity in women who delivered vaginally with an episiotomy and in those delivered without an episiotomy.

\section{METHODS}

Primigravid women were enrolled into the study on documentation of full dilatation of the cervix and randomized into either the Study Group (delivered without an episiotomy) or Control Group (delivered with an episiotomy). The objective of the study was to determine the occurrence of perineal morbidity in women who delivered vaginally with an episiotomy and in those who delivered without an episiotomy.

\section{Study design}

Randomized control study.

\section{Study population}

All women with singleton term pregnancies, fulfilling the inclusion criteria and planned for vaginal delivery (not requiring instrumental delivery) were enrolled into the study.

\section{Study period}

$1^{\text {st }}$ January 2013 to $31^{\text {st }}$ December 2013.

\section{Informed consent}

All the patients and the attenders gave written informed consent.

\section{Sample size calculation}

All primigravid women who underwent vaginal delivery (not requiring instrumental delivery) during the study period at JSS Hospital, Mysore and Maternity and Nursing Home, Siliguri were taken up for the study.

\section{Inclusion criteria}

All primigravidas with term $(37+$ weeks $)$ gestation, vertex presentation and singleton babies who were sure of their LMP with regular cycles [need to mention here practice in India involves unbooked patients with access to early pregnancy dating scans] or have their gestational age confirmed by USG [need to mention whether this was dating scan] and planned for vaginal delivery (not requiring instrumental delivery) irrespective of their age, height and weight.

\section{Exclusion criteria}

1. Multigravidas

2. Preterm babies

3. Multiple pregnancies

4. Instrumental delivery

5. Malpresentations

10 patients who were randomized into the study group, but required episiotomy due to foetal distress or poor maternal efforts were excluded from the study.

A detailed history taking, physical examination and obstetric examination followed by a fixed protocol for conduct of delivery was carried out for all parturients recruited for the study.

All labours were carefully monitored and if required were augmented with oxytocin (5 units oxytocin at 8 drops/min and increased by 8 drops/min every $30 \mathrm{~min}$ ). Women were in left lateral position during the 2 nd stage of labour and were allowed to bear down when they had the urge to do so. The standard time allowed for second stage of labour was 2 hours, if FHS was good. Care was taken to prevent perineal lacerations by, preventing too rapid an expulsion of the head by promoting flexion of the head in between contractions. Good perineal and para urethral support at the time of crowning of the head and during delivery of the baby was given either by two doctors (one supporting the perineum and para urethral area during delivery of head and shoulders and the other conducting the delivery). Oxytoxics were given within one minute of delivery of the baby.

Right mediolateral episiotomy was given for the control group. Placenta was delivered by Brandt Andrew's technique. Perineum was then examined in good light with patient in dorsal position and if any lacerations were noted it was sutured, if necessary, as per the institutional protocol (vicryl rapide was used). Episiotomy was sutured in 3 layers by the doctor who conducted the delivery with vicryl rapide. 


\section{RESULTS}

The total number of patients studied was 300 - equally distributed in both the groups. 10 patients who were randomized into the study group, but required episiotomy were excluded from the study. 6 patients required episiotomy due to foetal distress and the other 4 due to poor maternal efforts.

Statistical analysis was carried out using commercial software SPSS (Statistical Package for Social Sciences) Version 16. The descriptive measures like mean, median and standard deviation for continuous variables were obtained. Frequencies and percentages were calculated for all categorical variables.

The majority of patients in both the study group (without episiotomy) and control group (with episiotomy) were aged between 21-24 years and had their baby weights between 2.5 to $2.9 \mathrm{~kg}$.

In the study group, $22.0 \%(\mathrm{n}=33)$ patients had no lacerations in the perineum. Inspite of an episiotomy, $15.34 \%(n=23)$ had tears (anterior and posterior perineal lacerations) and in that $4.67 \%$ patients had anal sphincter tear. Out of the total of 18 patients with anal sphincter tear, 11 patients were from the study group (7.34\%) and 7 patients were from the control group (4.67\%). 9 patients had more than $50 \%$ of EAS torn (Category $3 \mathrm{~b}$ ) but none of them complained of incontinence during their followup.

Out of the total of the 300 patients only 220 patients could be followed up (103 in study group and 117 in the control group). 80 patients $(27 \%)$ could not be followed up as they either did not come back for their follow-up visit or could not be contacted over telephone. $68.37 \%$ (n $=80$ ) patients in the control group had persistent perineal pain for more than one week versus $19.42 \%(n=20)$ in the study group. No patients in the study group had pain more than 2 months while $4.27 \%$ patients $(n=5)$ in the control group had persistent pain.

The perineal morbidity in parturients who delivered without an episiotomy is definitely less than those who delivered with an episiotomy indicating that perineal pain is more frequent and severe for women with increased perineal trauma - like episiotomy.

\section{DISCUSSION}

The incidence of episiotomy in modern day obstetrics is approximately $81 \%$. There are wide variations in incidence of episiotomies among the different countries like, Netherlands report an episiotomy rate of $80 \%$, England \& Wales report 20\%, U.S.A report $50 \%$ and Eastern European countries report episiotomy rate as high as $99 \% .{ }^{4,5}$
Incidence of anterior perineal lacerations in my study was $6.0 \%$ (study group) in comparison to $10.67 \%$ (control group). The results differ from the observations made by Myers Helfgott et al and the Argentine Collaborative Trial that episiotomy decreases the anterior perineal lacerations. 6,7

The incidence of perineal trauma in the study group was $78.0 \%$ which was similar to the observation made by National Maternity Statistics, Department of Health, England (>85\% of woman who have vaginal birth will sustain some degree of perineal trauma). ${ }^{8}$ In our study, there were no 4th degree tears in both the groups. No perineal tears were noted in $22 \%$ of patients in the study group. These results were consistent with the study of Thacker \& Banta. ${ }^{9}$

Severe perineal lacerations- $3^{\text {rd }}$ degree perineal lacerations in my study was $7.34 \%$ (study group) in comparison to $4.67 \%$ (control group). 9 patients had more than $50 \%$ of EAS torn (Category $3 \mathrm{~b}$ ). Third-degree tears may be further subdivided into three subcategories ${ }^{10}$ :

1. 3a: partial tear of the external anal sphincter involving less than $50 \%$ thickness

2. 3b: greater than $50 \%$ tear of the external anal sphincter

3. $3 \mathrm{c}$ : internal sphincter is torn

Episiotomy does not protect the anal sphincter complex. Clinical judgement should dictate the necessity for an episiotomy. ${ }^{11}$ Episiotomy should be given only to patients where it is indicated. By doing so we can learn to be more patient and allow the natural forces of labour to gradually stretch the perineum. Severe perineal lacerations are associated with large babies, short $2^{\text {nd }}$ stage of labour, and lack of perineal support, rigid perineum and instrumental vaginal deliveries. ${ }^{12}$

WHO recommends that episiotomy rate should be around $10 \%$ and not more than $20 \% .^{13}$ The incidence of perineal pain after 1 week postpartum in my study was $19.42 \%$ (study group) versus $68.37 \%$ (control group), $\mathrm{p}<0.05$ (statistically significant). The perineal pain in patients with episiotomy (control group) even lasted for more than 2 months in 5 patients whereas no patients in the study group had such complaints. These above observations were consistent with the observation made by Macarthur AJ. ${ }^{14}$

\section{CONCLUSIONS}

This study throws light on the fact that short term perineal morbidity is significantly lower in parturients who delivered without an episiotomy and that episiotomy did not offer protection against sustaining severe perineal lacerations. Diverse rates of episiotomy in different countries suggest that the practice of episiotomy is not always justified. So, an attempt should be made to keep the incidence of episiotomy as low as possible. It is 
difficult to obtain a global perspective on spontaneous perineal trauma requiring suturing, due to inconsistency in classification and under reporting of perineal trauma. ${ }^{15}$ Changing the way obstetricians practice can be difficult. Ideally a dedicated "Perineal Dysfunction Clinic" should be set up, for follow up of women experiencing persistent problems after delivery, consisting of an obstetrician and physiotherapist.

Funding: No funding sources

Conflict of interest: None declared

Ethical approval: The study was approved by the Institutional Ethics Committee

\section{REFERENCES}

1. Sleep J, Grant A, Garcia J, Elbourne D, Spencer J, Chalmers I. West Berkshire - Perineal Management Trial. BMJ. 1984;289(6445):587-90.

2. Sreevidya S, Sathiyasekaran BW. High caesarean rates in Madras (India): A population-based cross sectional study. BJOG. 2003;110(2):106-11.

3. Jeffrey L. Ecker, Winona M. Tan, Raj K. Bansal, Judith T. Bishop, Sarah J. Kilpatrick. Is there a benefit to episiotomy at operative vaginal delivery? Observations over 10 years in a stable population. Am J Obstet Gynecol. 1997;176(2):411-4.

4. Renfrew MJ, Hannah W, Albers L, Floyd E. Practices that minimise the trauma to the genital tract in child birth: A systematic review of the literature. Birth. 1998;25(3):143-60.

5. Graves EJ. Summary: National hospital discharge survey. Advance data from vital health statistics, 1995.

6. Myers-Helfgott MG, Helfgott AW. Routine use of episiotomy in modern obstetrics: Should it be performed? Obstet Gynecol Clin North Am. 1999;26(2):305-25.
7. Belizan J, Campodonico L, Carroli G, Gonzalez L, Lede R. - Argentine Collaborative Trial. Routine vs selective episiotomy: A randomized controlled trial. Lancet. 1993;342(8886-7):1517-8.

8. Alison Macfarlane. At last maternity statistics for England- Department of Health. BMJ. 1998;316(7131):566-7.

9. Thacker SB, Banta HD. Benefits \& risks of episiotomy: An interpretive review - 1860-1980. Obstet Gynecol Surv. 1983;38(6):322-38.

10. De Leeuw JW, Struijk PC, Vierhout ME, Wallenburg HCS. Risk factors for 3rd degree perineal ruptures during delivery. BJOG. 2001;108(4):383-7.

11. Angioli R, Gomez-Marin O, Cantuaria G. Severe perineal lacerations during vaginal delivery: The University of Miami experience. Am J Obstet Gynaecol. 2000;182:1083-5.

12. Deering SH, Carlson N, Stitely M, Allaire AD, Satin AJ. Perineal body length and lacerations at delivery. J Reprod Med. 2004;49(4):306-10.

13. Fernando RJ, Sultan AH, Kettle C, Radley S, Jones $\mathrm{P}, \mathrm{O}$ 'Brien PM. Repair techniques for obstetric anal sphincter injuries: A randomized controlled trial. Obstet Gynaecol. 2006 Jun;107(6):1261-8.

14. Macarthur AJ, Macarthur C. Incidence, severity \& determinants of perineal pain after vaginal delivery: A prospective cohort study. Am J Obstet Gynecol. 2004;191(4):1199-204.

15. Martin S, Labrecque M, Marcoux $S$, Berube S, Pinault JJ. The association between perineal trauma and spontaneous perineal tears. J Fam Pract. 2001;50(4):333-7.

Cite this article as: Roy P, Sujatha MS, Biswas B, Chatterjee A, Roy P. A comparative study of perineal morbidity in vaginal delivery with and without episiotomy. Int $\mathbf{J}$ Reprod Contracept Obstet Gynecol 2015;4:1442-5. 